\title{
Nephrotic Syndrome with Lesion of Hypocomplementemic Glomerulonephritis
}

National Cancer Institute

\section{Source}

National Cancer Institute. Nephrotic Syndrome with Lesion of Hypocomplementemic

Glomerulonephritis. NCI Thesaurus. Code C35536.

Nephrotic syndrome caused by glomerulonephritis associated with complement deficiency. It can result from an acute post-infectious glomerulonephritis, membranoproliferative glomerulonephritis or lupus glomerulonephritis. 